\title{
Viscoelastic monitoring of starch-based biomaterials in simulated physiological conditions
}

\author{
J.F. Mano ${ }^{a, b, *}$ R.L. Reis ${ }^{a, b}$ \\ ${ }^{a}$ Department of Polymer Engineering, Campus de Azurém, University of Minho, Guimarães 4800-058, Portugal \\ b 3B's Research Group-Biomaterials, Biodegradables and Biomimetics, Campus de Gualtar, University of Minho, Braga 4710-057, Portugal
}

Received 12 July 2002

\begin{abstract}
Dynamic mechanical analysis (DMA) was used to investigate the solid-state rheological behaviour in a starch-based thermoplastic aimed to be used in different biomedical applications. The tested samples were processed by different injection moulding procedures. The dry samples were immersed in a simulated physiological solution and the relevant viscoelastic parameters were monitored against time. The decrease of stiffness due to swelling can be followed in real time, being less pronounced for the composite sample with hydroxyapatite (HA). The temperature control of the liquid bath was found to be very good. Frequency scans were also performed in wet conditions in samples previously immersed during different times, indicating that DMA is a suitable method to control in-vitro the changes on the viscoelastic properties of biomaterials during degradation.
\end{abstract}

(C) 2003 Published by Elsevier B.V.

Keywords: Thermal analysis; Biodegradable; Dynamic mechanical behaviour; Natural polymers

\section{Introduction}

Recently, different starch/synthetic polymer blends, have been shown to have potential to be used in distinct biomedical applications. These include their use as scaffolds for the tissue engineering of bone and cartilage [1], materials for bone fixation and replacement as well as for filling bone defects [2,3], carriers for the controlled release of drugs and other bioactive agents [4], and new hydrogels and partially degradable bone cements [5,6]. An intense research program is in progress that pretends to fully characterise such materials in all relevant vectors, such as biological response, surface characteristics, mechanical and thermal properties, swelling, degradation behaviour, ability for chemical modifications, etc.

Living tissues and, obviously, their biological constituents (such as bone, cork, wood, starch, living cells, ...), present a viscoelastic behaviour near room (or body temperature) and appropriate time scales (e.g. $1 \mathrm{~Hz}$ ), suggesting that natural materials are able to dissipate certain amounts of me-

\footnotetext{
* Corresponding author. Tel.: +35-253-510-245; fax: +35-253-510-249.

E-mail address: jmano@dep.uminho.pt (J.F. Mano).
}

chanical energy [7]. Thus, it is important the characterisation of the viscoelastic of novel materials (aimed to mimic nature) to be used in biomedical applications. Moreover, in most situations an implanted device is in a solid or gel phase and subjected to complex stress fields. This is the typical situation for implants to be used on substitution or regeneration of bone, dental deficiencies, in maxilofacial surgery or in cardiovascular grafts and devices. As polymeric systems are viscoelastic in their own nature, the knowledge of their solid-state rheological behaviour is very important in the prediction of their mechanical performance in a real service situation.

This characterisation would be more efficient if the products are tested under close physiological conditions, i.e. immersed in simulated physiological fluids and at body temperature. Dynamic mechanical analysis (DMA) is a non-destructive technique widely used in the characterisation of polymer-based systems, which is getting increasing importance in biomaterials research. In some previous studies, DMA has proved to be suitable to test immersed samples, such as textile fibres or films [8,9] or hydrogels [10]. This work pretends to demonstrate that DMA experiments in-vitro may be performed in a starch-polymer blend system and that informations concerning the mechanical/damping 
behaviour, swelling and degradation may be extracted both from on-line and off-line procedures.

\section{Experimental}

\subsection{Apparatus}

The dynamic mechanical analysis experiments were carried out in a DMA7e Perkin-Elmer apparatus, using the three-point bending mode, where bending platforms of 15 and $20 \mathrm{~mm}$ were used. The real (storage) modulus, $E^{\prime}$, and the imaginary (loss) modulus, $E^{\prime \prime}$, components of the complex modulus, $E^{*}=E^{\prime}+\mathrm{i} E^{\prime \prime}$ were recorded against time, for a fixed frequency of $1 \mathrm{~Hz}$, or against frequency, that varied between 0.5 and $10 \mathrm{~Hz}$. The amplitudes of the dynamic loads used were small enough to ensure a linear viscoelastic response of the samples.

For the tests of the immersed samples, a cylindrical metallic vessel with $0.5 \mathrm{~mm}$ wall thickness was fitted in the furnace of the equipment, taking the advantage of the vertical assembling of the apparatus pieces (furnace, probe tip, etc., $\ldots$... The out-side diameter of the cylindrical vessel was as much as possible close to the inner-side diameter of the furnace cavity, in order to enhance the thermal contact between the furnace and the bath. The vessel was filled with isotonic saline solution $(0.154 \mathrm{M} \mathrm{NaCl}$ aqueous solution at $\mathrm{pH} 7.4)$.

The calibration of the force of the apparatus was performed using a $50 \mathrm{mg}$ weight, with the probe tip immersed, which took into account the impulsion effect of the solution. In fact, prior experiments on non-degradable and hydrophobic polyethylene samples (injection moulded pieces with rectangular cross section) indicated that the storage modulus, $E^{\prime}$, could depend on the liquid level. The viscous drag and the inertial effect of the fluid of the experiment may also affect the measurements of the sample $\tan \delta$. This kind of analysis was done by Dillman and Seferis [11], using classic dimensional analysis [11]. It was found that storage and loss friction parameters could be determined and subtracted from the measured results to provide fluid-independent dynamic mechanical properties of the sample [11]. We found, however, that the $\tan \delta$ of an hydrophobic elastomer measured in dry or wet conditions (under the same experimental settings) was very similar, indicating that at a first analysis such corrections could be negligible.

The temperature of the bath was stabilised at $37^{\circ} \mathrm{C}$ prior to the runs on the samples, using the own temperature sensor of the apparatus.

\subsection{Materials}

The studied material was a blend of starch (around $50 \pm$ $2 \mathrm{wt} . \%)$ and a copolymer of ethylene and vinyl alcohol $(60 / 40(\mathrm{~mol} / \mathrm{mol}))$ designated as SEVA-C. A particulate synthetic hydroxyapatite (HA) supplied by Plasma Biotal (UK), was used as reinforcement. The ceramic reinforcement has an average particle size of $10.1 \mu \mathrm{m}$.
SEVA-C was processed by injection moulding into tensile tests specimens with circular cross section ( $5 \mathrm{~mm}$ diameter). Two injection moulding approaches were used: conventional (CM) and shear controlled orientation in injection moulding (SCORIM). In SCORIM processing the molten material, after filling the mould cavity, is forced to move between two piston chambers, inducing a macroscopic shear stress field at the melt-solid interface during the solidification process. The obtained materials show higher levels of anisotropy and improved mechanical properties as compared with the CM ones. SEVA-C was also compounded with $30 \mathrm{wt} . \%$ of HA in an extruder in order to obtain composite samples, by processing with SCORIM. More details about the processing of these materials may be found elsewhere [3].

Complementary studies were also carried out with thermoplastic potato starch samples (rectangular cross section of ca. $\sim 2 \mathrm{~mm} \times 5 \mathrm{~mm}$ ) processed by $\mathrm{CM}$.

\section{Results and discussion}

A degradable biomaterial in contact with tissues will experiment humid environment, at $37^{\circ} \mathrm{C}$. The mechanical properties will change with time due to different factors, such as swelling or degradation. DMA provides a way to follow in real time the rheological changes in in vitro conditions. The first example of such tests will be given with the dry potato starch samples (Fig. 1), where the storage modulus is monitored as a function of time just after immersion (triangles) and in dry conditions (squares). The storage modulus of all samples start at the same point but the immersed ones begins immediately to lose stiffness, as swelling proceeds quickly. Two different samples were used (up and down triangles), the respective results being very reproducible. At $t \sim 90 \mathrm{~min}, E^{\prime}$ is very small and the samples contact with the bottom of the bending platform and the experiment must be considered finished. Such experiments show that DMA may be used to follow the mechanical properties during swelling just after few seconds of immersion, being an advantage in materials having a fast swelling rate. Fig. 1 shows that during the dry experiment we do not have an horizontal trend (the stabilisation of $E^{\prime}$ occurs after $10 \mathrm{~min}$ ) that may be caused by the adaptation of the sample to the new environment with a different moisture level. More details on the viscoelastic properties of the studied starch in the dry state may be found elsewhere [7].

The viscoelastic properties of SEVA-C, in dry conditions, were already reported in previous papers $[12,13]$. However, no in-situ and in vitro testing has been reported before. CM samples were tested by DMA and two relaxation processes were seen in the temperature region between -20 and $130^{\circ} \mathrm{C}$ [12], assigned to molecular motions within the copolymer phase (at $\sim 30^{\circ} \mathrm{C}$ ) and within the starch phase (at $\sim 90^{\circ} \mathrm{C}$ ). The former relaxation shifts to lower temperature with increasing low moisture content, as a result of a plasticisation 


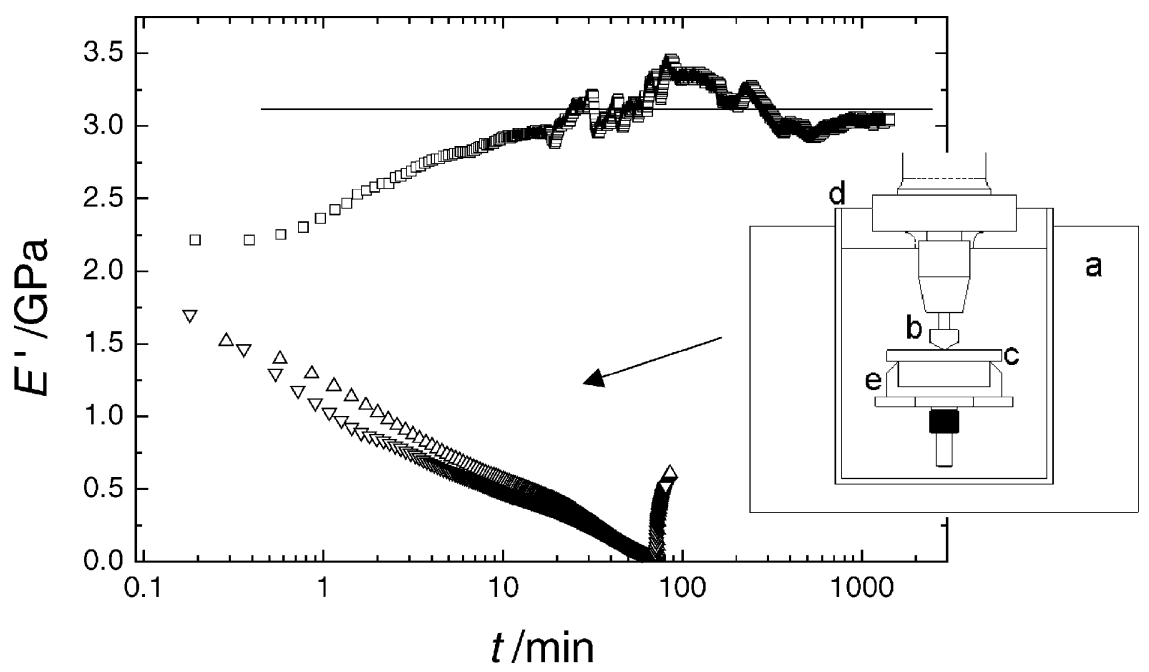

Fig. 1. Storage modulus at $1 \mathrm{~Hz}$ against time performed in dry potato starch samples. Squares: The measurements are carried out in dry conditions; the values stabilise around $3 \mathrm{GPa}$ (solid line). Triangles: experiments on two samples in wet conditions (the dry sample is immersed at $t=0$ min); note that no geometrical correction was performed in such values, assuming that the geometry of the samples (cross-section dimensions) was time invariant. The $\log$ scale permits to amplify the smaller time region. The scheme on the right shows the relevant parts of the set-up that allows to perform experiment under liquid environments: (a) DMA furnace; (b) probe-tip; (c) sample; (d) container with simulated physiological solution and (e) bending platform.

effect of water [12]. The introduction of HA in CM SEVA-C samples was also investigated by DMA [13], and the combination of HA and SCORIM processing was also studied in dry samples [3].

Tests in immersion at physiological conditions were performed in the SEVA-C samples, initially in the dry state, at $37^{\circ} \mathrm{C}$. Particular attention was paid for the correction of the storage modulus that took into account the change of the diameter of the samples due to swelling. In complementary experiments the diameter of the samples was measured as a function of time (inset in Fig. 2a), which could be used in such corrections. The time dependence of the diameter, $d$, was fitted by the empirical function: $d=a+b t^{c}$, being $a, b$ and $c$ adjustable parameters (solid lines in the inset graphics in Fig. 2a); this best fitting equation was automatically found among 99 simple equations using the TableCurve $2 \mathrm{D}^{\mathrm{TM}}$, Version 4, from Jandel scientific software.

The uncorrected and corrected $E^{\prime}$ versus time results are shown in Fig. 2a. As for pure starch, $E^{\prime}$ decreases due to the swelling of the samples. However, the swelling kinetics of the blends is much slower than for pure starch (Fig. 1) due to the presence of the hydrophobic synthetic copolymer. It was found, in fact, that the diameter of the samples stabilised after $\sim 1$ week, up to values less than $10 \%$ higher than that of the initial values. In this context, it should be noticed that the water absorption kinetics of such samples may be changed by chemical modification, such as cross-linking [14] or by grafting hydrophobic groups.

The storage modulus of the SCORIM processed sample was found to be always higher than the CM one, indicating that this processing technique maintains the mechanical stiffness of the samples even when they are in liquid environments. As expected, the introduction of HA increases further $E^{\prime}$, with respect to the matrix (Fig. 2a).
The variation of the damping properties were also followed against time in the SEVA-C samples after immersion. This is an important parameter to be monitored as it will determine the capability of the material to absorb mechanical energy when the implant is subjected to periodic loads with equivalent frequencies of about $1 \mathrm{~Hz}$. Fig. $2 \mathrm{~b}$ shows that in all samples $\tan \delta$ decreases with time, being a consequence of the absorption of the solution. This can be due to two reasons: the plasticisation of water shifts the relaxation at $-30{ }^{\circ} \mathrm{C}$ to lower temperatures and thus $\tan \delta$ decreases (at $37^{\circ} \mathrm{C}$ the characteristic times of the relaxation are too high) or, on the other hand, the viscous nature of water may interfere itself on the overall viscoelastic of the system. A system composed by a viscoelastic element (SEVA-C), with a complex modulus of $E_{\mathrm{VE}}^{*}$, coupled in series with a viscous element, with $E^{*}=\mathrm{i} \omega \eta$, exhibits a final modulus of $E^{*}=$ $\left(x / E_{\mathrm{VE}}^{*}+(1-x) / i \omega \eta\right)^{-1}$, where $x$ is the volume fraction of SEVA-C, $\omega$ is the angular frequency and $\eta$ is the viscosity of the viscous element. It can be shown that as $x$ decreases, i.e. as swelling proceeds, the loss factor of this system, $\tan \delta$, decreases. The results in Fig. 2b also suggest that the loss factor of SEVA-C depends weakly on the processing procedure used (CM or SCORIM), although Fig. 2a showed that the stiffness is higher for the SCORIM sample. However, it seems that the incorporation of HA leads to samples with higher damping capabilities. This behaviour could be explained by the energy lost in the interface between the matrix and the ceramics, due to macroscopic frictional effects in this heterogeneous system. The true loss modulus $\left(E^{\prime \prime}\right)$ was also monitored against time (inset graphics in Fig. 2b). It decreases with increasing time, more quickly for the $\mathrm{CM}$ sample and with higher values for the SEVA-C/HA sample. This is a consequence of the same trends observed in $E^{\prime}$ $\left(E^{\prime \prime}=E^{\prime} \tan \delta\right)$. 

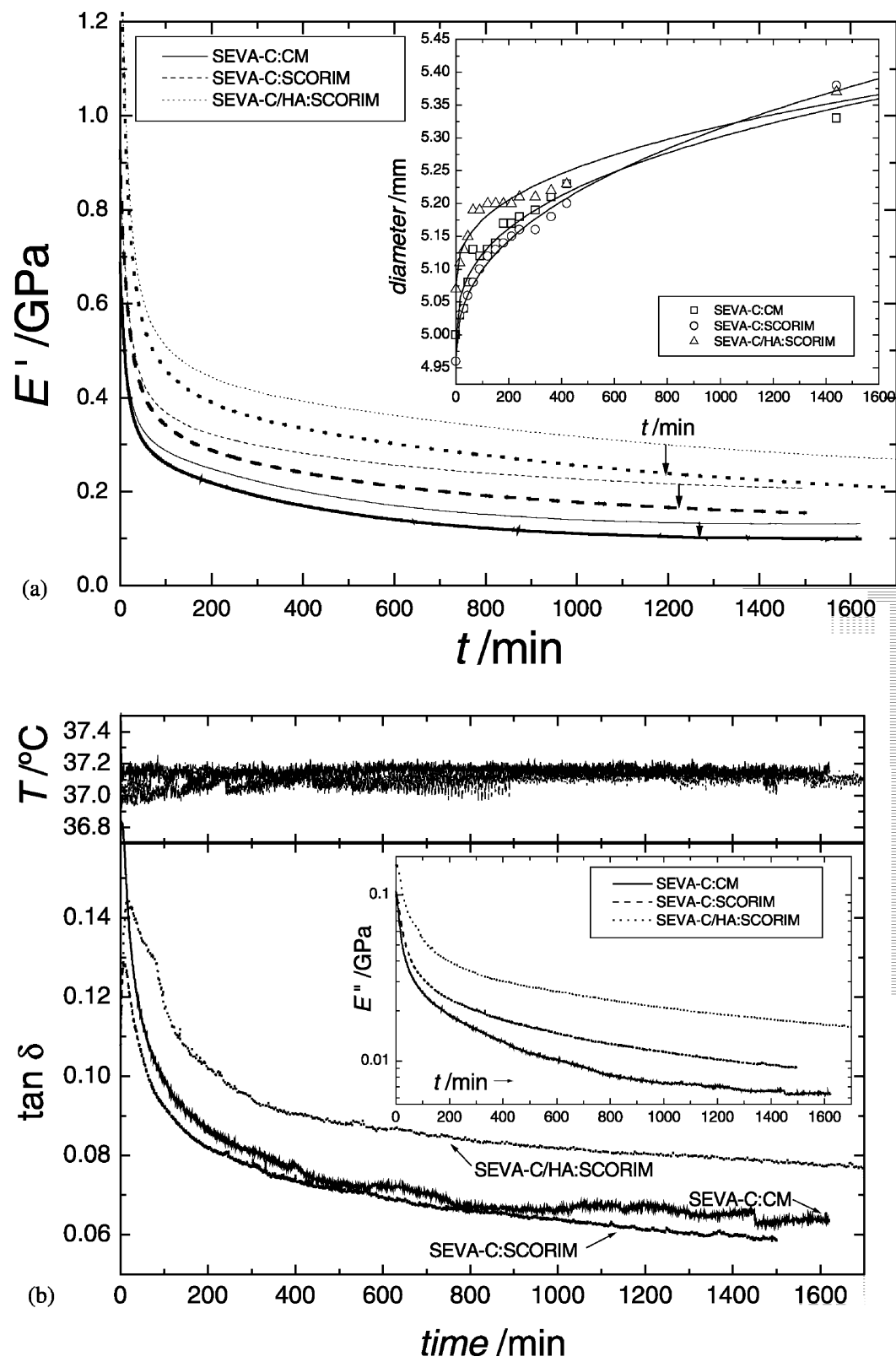

Fig. 2. Immersion experiments on SEVA-C-based samples. (a) Time dependence of the storage modulus. The thinner lines are for $E^{\prime}$ measured assuming a constant diameter. The thicker lines assumed a correction due to the change of the diameter of the samples, obtained from off-line measurements of the diameter as a function of time (symbols in the inset graphics), that were fitted by the empirical function: diameter $=a+b t^{c}$, being $a, b$ and $c$ adjustable parameters (solid lines). The arrows link the uncorrected curves to the corrected ones, for each material. (b) The bottom graphics shows the time dependence of the loss factor $\left(\tan \delta\right.$ ) of the materials after immersion (the inset graphics shows the time dependence on the loss modulus $E^{\prime \prime}$, obtained from the corrected $E^{\prime}$ shown in (a), $E^{\prime \prime}=E^{\prime} \tan \delta$ ); the top graphic shows the temperature read by the sensor during the experiments on immersion.

The implementation of mechanical tests of biomaterials in immersion should imply a good temperature control around $37^{\circ} \mathrm{C}$. Thus, the temperature read by the apparatus sensor was also monitored against time (top graphics in Fig. 2b) for the three experiments reported. An excellent agreement was found between the different runs, indicating a good reproducibility of the temperature reading. Moreover, the temperature was found to fluctuate less than $0.2^{\circ} \mathrm{C}$ in the whole time range. The liquid mass in the vessel is thus sufficiently small so that the DMA furnace is able to accurately control its temperature changes. The mean temperature is slightly higher than $37^{\circ} \mathrm{C}$ but, by changing the temperature set-up, it could be closer to $37^{\circ} \mathrm{C}$. This results show that an excellent temperature control can be achieved in this kinds of experiments.

This work aims also to point out that DMA is a suitable method to follow off-line the degradation behaviour of biomaterials for long time periods. The samples are externally 


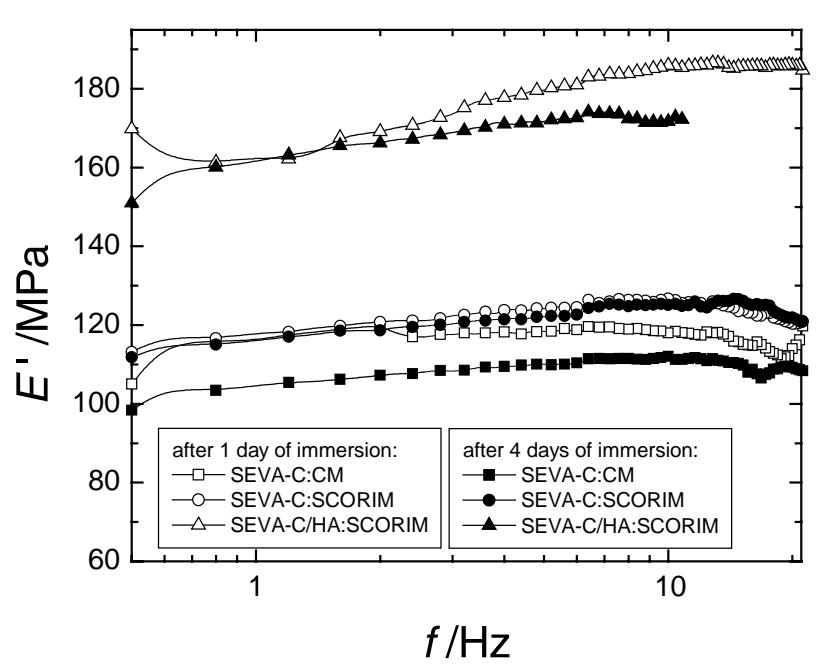

Fig. 3. Storage modulus vs. frequency for the three studied samples performed in wet conditions and $37^{\circ} \mathrm{C}$ after 1 day (open symbols) and 4 days (filled symbols) of immersion in simulated physiological solution at $37^{\circ} \mathrm{C}$.

immersed in a $37^{\circ} \mathrm{C}$ simulated physiological solution and periodically tested in the DMA apparatus. Eventually, the same sample may be tested at different times, as this technique provides a non-destructive way to monitor its mechanical performance. As an example, Fig. 3 shows $E^{\prime}$ versus frequency for the three studied samples measured in wet conditions after 1 and 4 days of immersion. Obviously, $\tan \delta, E^{\prime \prime}$ or other viscoelastic parameter could also be plotted. Extended degradation times could also be shown; however, it was found that after 4 days the $E^{\prime}$ versus $f$ values for each sample stabilised within the experimental errors (up to 30 days). These finding are in accordance with previous DMA results obtained in dry samples, were no significant decreasing of stiffness was found up to 60 days [12]. The weight loss of SEVA-C samples was also found to be quite stable up to 30 days, after the second day of immersion on an isotonic saline solution [14].

The $E^{\prime}(f)$ plot in Fig. 3 for a given sample is found to be quite horizontal, suggesting that in these temperature and frequency regions no relaxation process takes place. This means that implanted pieces obtained from such materials should exhibit similar viscoelastic properties whatever the time scale of the external mechanical perturbation (for example running or walking).

For each immersion time, the stiffness of the composite is always higher than the matrix and the CM samples tends to present the lowest $E^{\prime}$ values, in the whole frequency range (Fig. 3). The increase of the immersion time (from 1 to 4 days) seems to decrease $E^{\prime}$, which may still be a consequence of the swelling, which may proceed beyond $24 \mathrm{~h}$.

\section{Conclusions}

This work demonstrated that the viscoelastic properties of a series of starch-based biomaterials could be studied by
DMA under in vitro conditions, i.e. immersed in a simulated physiological solution at controlled body temperature. It was shown that the relevant viscoelastic parameters of the samples can be monitored as a function of time or frequency. Such experiments allows for a more reliable description of the mechanical performance of biomaterials in service conditions.

The viscoelastic properties in the studied systems were found to be dependent on the processing conditions of the samples. In particular, the inclusion of hydroxyapatite particles and the use of non-conventional injection moulding techniques (that enhances the sample's anisotropy) increase both the stiffness and the damping properties of the samples in wet conditions.

The mechanical properties of biodegradable systems are changing during degradation, which may be followed, for example, as a function of frequency, for different degradation times, using the proposed procedure. This may be a consequence of swelling or degradation (chain scission and low molecular mass leaching). Again, one may conclude that the used method helps in the non-destructive monitoring of the mechanical performance evolution in biomaterials during degradation.

\section{Acknowledgements}

The authors thank Dr. Rui Sousa for the aid in the injection moulding of the samples.

\section{References}

[1] M.E. Gomes, A.S. Ribeiro, P.B. Malafaya, R.L. Reis, A.M. Cunha, Biomaterials 22 (2001) 883.

[2] R.L. Reis, A.M. Cunha, J. Appl. Med. Polym. 4 (2000) 1.

[3] R.A. Sousa, J.F. Mano, R.L. Reis, A.M. Cunha, M.J. Bevis, Polym. Eng. Sci. 42 (2002) 1032.

[4] P.B. Malafaya, C. Elvira, A. Gallardo, J. San Román, R.L. Reis, J. Biomat., Sci. Polym. Ed. 12 (2001) 1227.

[5] C. Elvira, J.F. Mano, J. San Román, R.L. Reis, Biomaterials 23 (2002) 1955.

[6] I. Espigares, C. Elvira, J.F. Mano, B. Vasquez, J. San Roman, R.L. Reis, Biomaterials 23 (2002) 1883.

[7] J.F. Mano, R.L. Reis, A.M. Cunha, in: R.L. Reis, D. Cohn (Eds.), Polymer-based Systems on Tissue Engineering, Replacement and Regeneration, Nato Science Series, Kluwer Academic Publishers, Dordrecht, 2002.

[8] A.B. Desai, G.L. Wilkes, Text. Res. J. 45 (1975) 173.

[9] H.A. Davis, J. Text. Inst. 86 (1995) 332.

[10] Q.-B. Bao, C.S. Bagga, Thermoch. Acta 226 (1993) 107.

[11] S.H. Dillman, J.C. Seferis, Polym. Eng. Sci. 31 (1991) 253.

[12] J.F. Mano, R.L. Reis, A.M. Cunha, J. Appl. Polym. Sci. 78 (2000) 2345.

[13] J.F. Mano, C.M. Vaz, S.C. Mendes, R.L. Reis, A.M. Cunha, J. Mater. Sci.: Mater. Med. 10 (1999) 857.

[14] D. Demirgös, C. Elvira, J.F. Mano, A.M. Cunha, E. Piskin, R.L. Reis, Polym. Degrad. Stab. 70 (2000) 161. 